\title{
2016 closure
}

\author{
Yoram Reich ${ }^{1}$
}

Published online: 4 January 2017

(C) Springer-Verlag London 2017

Following 2015, 2016 has been again a successful year for Research in Engineering Design. We end a year with a record high of submissions: over 220 until the writing of this editorial. We also end the year with a backlog of 11 papers waiting to be published. This reflects an increase in the quality submissions that are accepted compared to previous years. The management of the review process seems to be more streamlined but this is an aspect that can always be further improved.

None of this would have been possible without the extended community supporting the journal: the authors that trust the results of their research with the journal team, the area editors and the editorial board members, and the many dedicated reviewers. I would like to extend my deep gratitude go to the numerous reviewers who contributed their effort to maintain and improve the high quality of the journal. The reviewers, outside the editorial board, who completed a review this year, are:

\author{
Aggeri Franck \\ Agogue Marine \\ Ahmad Naveed \\ Allen Janet \\ Allison James \\ Annamalai Vasantha Gokula Vijaykumar \\ Antani Kavit \\ Aurisicchio Marco \\ Austin-Breneman Jesse \\ Balamurugan $\mathrm{C}$. \\ Barnes Catherine \\ Billington Scott \\ Biswas Pradipta \\ Blanco Eric \\ Blessing Lucienne \\ Bos Bram \\ Bouchard Carole \\ Boujut Jean Francois
}

Yoram Reich

yoram@eng.tau.ac.il

1 School of Mechanical Engineering, Tel Aviv University, Tel Aviv, Israel
Brown David

Browning Tyson

Caldwell Nicolas

Campbell Matthew

Cardin Michel-Alexandre

Cavallucci Denis

Chang Hua-Cheng

Chen Aoyu

Chen Wei

Chulvi Vicente

Ciucci Francesco

Claudet Andre

Coatanéa Eric

Colombo Edoardo

Crilly Nathan

De Guio Roland

De Lessio Mark

de Weck Oliver

Deken Fleur

Dekoninck Elies

Dinar Mahmoud

Dori Dov

Duhovnik Joze

DuPont Bryony 
Eisenbart Boris

Esparragoza Ivan

Eynard Benoit

Farid Amro

Franssen Maarten

Freitas Salgueiredo Camila

Fu Wentao

Fu Katherine

Fukuda Shuichi

Germani Michele

Giunta Anthony A.

Goh May

Grogan Paul

Guariniello Cesare

Hansen Claus

Hatchuel Armand

Hendriks Lex

Henson Brian

Hernandez Alberto

Holtta-Otto Katja

Honda Tomonori

Horvath Imre

Howard Thomas

Huang George

Imai Tomoko

Isaksson Ola

Jagtap Santosh

Jankovic Marija

Jiao Jianxin Roger

Juuti Tero

Kang Namwoo

Keinonen Turkka

Kelly Nick

Kim Sang-Gook

Kipouros Timoleon

Kirchner Eckhard

Kleinsmann Maaike

Koh Edwin

Kokshagina Olga

Kroll Ehud

Lauche Kristina

Ledoux Yann

Legardeur Jeremy

Levillain Kevin

Lewis Kemper

Li Simon

Li Zhizhong

Linsey Julie

Liu Cong

Liu Ying-Chieh

Ma Yongsheng

MacDonald Erin

Magnin Michael
Maier Anja

Malak Jr. Richard

Marle Franck

Mattson Christopher

McGilp Helen

McManus Hugh

Mears Laine

Meijer Sebastiaan

Mink Annemarie

Mocko Greg

Montagna Francesca

Moon Seung Ki

Morkos Beshoy

Moullec Marie-Lise

Mulet Elena

Nath Chandra

Nieusma Dean

Nordlund Mats

Otto Kevin

Panchal Jitesh

Park Yong Seok

Parraguez Ruiz Pedro

Peng Qingjin

Polini Wilma

Qamar Ahsan

Rodgers Lennon

Rodriguez Edgar

Ross Adam

Sacks Rafael

Salhieh Sa'ed

Salustri Filippo

Sarkar Prabir

Sarkar Biplab

Scott Michael

Seepersad Carolyn

Sen Dibakar

Sen Chiradeep

Shea Kristina

Shu Lily H.

Siddique Zahed

Slocum Alex

Sobek, II Durward

Steinert Martin

Storto Corrado

Summers Joshua

Tahera Khadija

Tate Derrick

Thompson Mary Kathryn

Thompson Avril

Thurston Deborah

Tsoukias Alexis

Tucker Conrad

Tyagi Satish 
Ullah AMM Sharif

Ullman David

van Eck Dingmar

Van Heerden Stevan

Vargas Hernandez Noe

Venkataraman Srinivasan

Vourch Gwenael

Warell Anders

Weixelbaum Ilona

Whitney Daniel
Wood Kristin L.

Wynn David

Xu Qianli

Xue Deyi

Yang Maria

Yi-Min Deng

Yoshioka Masaharu

Zeng Yong

Zolghadri Marc 